\title{
Uc.206 regulates cell proliferation and apoptosis by targeting P53 in cervical cancer cells
}

\author{
Q. $\mathrm{LI}^{1}, \mathrm{X} . \mathrm{LI}^{2}, \mathrm{C} . \mathrm{WANG} \mathrm{W}^{2,3,4, *}$
}

${ }^{1}$ Medical College of Yangzhou Polytechnic University, 458 Wenchangxilu Yangzhou, 225009, China; ${ }^{2}$ Department of Pathology, Yangzhou University School of Medicine, 11 Huaihai Road, Yangzhou, 225001, China; ${ }^{3}$ Jiangsu Key Laboratory of Integrated Traditional Chinese and Western Medicine for Prevention and Treatment of Senile Disease; ${ }^{4}$ Jiangsu Co-Innovation Center for Prevention and Control of Important Animal Infectious Disease and Zoonoses, 11 Huaihai Road, Yangzhou 225001, China

*Correspondence: 429350063@qq.com

Received October 17, 2015 / Accepted January 11, 2016

\begin{abstract}
Ultraconserved regions (UCRs) are non-protein coding gene sequences with strict conserved across among different species. Emerging evidence demonstrates that UCRs encoding noncoding RNAs (ncRNAs) serve as regulators of gene expression. In recent decades, increasing evidence implicates the involvement of UCRs in carcinogenesis. Previous studies showed RNA expression of uc.206 was increased in colorectal cancer. Until now, the role of uc.206 in cervical cancers remains undefined. This study revealed that uc.206 is significantly up-regulated in cervical cancer (CC) tissue and negatively correlates with the expression of the pro-apoptotic gene P53 in RNA level. We show that uc.206 specifically targets the 3' untranslated region (3'UTR) of P53 and regulates its expression. Inhibition of uc.206 effectively delays cervical cells proliferation and promotes apoptosis, accompanied by increased expression of P53 protein. Thus, these findings suggested that uc.206 acts as a novel oncogene by targeting the P53 gene and promoting CC cell growth, which might be beneficial for cervical cancer therapy.
\end{abstract}

Key words: uc.206, P53, proliferation, apoptosis, cervical cancer

Cervical cancer is the second most common cause of female specific cancer after breast cancer and the fourth leading cause of cancer mortality in women worldwide (1). Approximately $80 \%$ of cervical cancers occur in developing countries (2).

Because detection of precursors by cytological examination and their eradication by laser vaporization or cone biopsy is the most effective method of cervical cancer prevention, the carcinoma is very treatable, and in many cases, curable. However, once this cancer grows again and spreads to the distant lymph nodes, the outlook is fatal in advanced carcinoma (3). Therefore, it is essential to identify proliferation-associated molecules and to better understand the mechanisms behind the growth of cervical carcinoma.

Ultraconserved regions (UCRs) are noncoding gene sequences with strict conserved across among mice, rats, and humans. It belongs to part of non-protein-coding DNA and regulates expression and translation of mRNAs. Emerging evidence demonstrates that UCRs can encode non-protein-coding RNAs (ncRNAs) serve as modulators of gene expression $(4,5)$.
Previous genome expression profiling studies showed that transcribed ultraconserved RNAs (T-UCRs) exhibit different profiles in human carcinomas $(6,7)$. Moreover, some T-UCRs serve as oncogenes or tumor suppressor genes in development of tumor. For example, the transcribed ultraconserved element 73 (uc.73) modulates cell proliferation in colorectal cancer cell lines (6). However, the regulation of most T-UCRs and their precise mechanisms of action are not known in cervical carcinoma.

Uc.206 is one of T-UCRs. Calin GA, et al. studies indicated that RNA expression of uc. 206 was up-regulated in colorectal cancer (6). However, little is known about the expression level and biological role of uc.206 in cervical carcinoma.

In the presented study, the differential expression of uc.206 in human CC samples was identified using quantitative polymerase chain reaction (qPCR), and the function of uc.206 in proliferation and apoptosis of cervical cancer cells was investigated. In addition, to understand the growth mechanism of cervical cancer, the target gene of uc.206 was further investigated. To the best of our knowledge, this is the first study to 
investigate the expression and mechanism of uc. 206 in cervical carcinoma proliferation. This study presents a novel target for further therapeutic studies of cervical cancer.

\section{Materials and methods}

CC tissue collection. A total of 40 paired tissue specimens from CC and matched normal tissue were used in this study and obtained from 40 CC patients (age range, 40-68 years) at the Departments of Obstetrics and Gynecology of the Jiangsu Subai People Hospitals, affiliated to Yangzhou University (Yangzhou, China). The absence of tumor cells in the matched normal tissues was confirmed by a pathologist. All tissues were obtained during surgery and immediately stored in liquid nitrogen prior to use. Approval for this study was granted by the Institute Research Medical Ethics Committee of the Medical College of Yangzhou University (Yangzhou, China). Patients provided written informed consent.

Cell line culture. Human cervical cancer cell lines (Hela and C33A) and 293T cell line were purchased from the Chinese Peking Union Medical College Cell Bank (Beijing, China). All cell lines were maintained in DMEM and RPMI 1640 supplemented with $10 \%$ fetal bovine serum (FBS) (HyClone Victoria, Australia), $100 \mathrm{IU} / \mathrm{mL}$ penicillin, and 100 $\mathrm{mg} / \mathrm{mL}$ streptomycin at $37^{\circ} \mathrm{C}$ in a humidified atmosphere containing $5 \% \mathrm{CO}_{2}$.

Plasmids. The uc.206-murine stem cell virus (MSCV) plasmid and migRI-P53-GFP plasmid were chemically synthesized at the Department of Pathology, Medical College of Yangzhou University (Yangzhou, Jiangshu) and sequenced by Sangon Biotech (Shanghai) Co., Ltd. (Shanghai, China). The uc.206 inhibitor and the scramble control were purchased from Sangon Biotech. shRNA that specific to human P53 (TGCGTGTGGAGTATTTGGATG) and its scrambled control were synthesized by Sangon Biotech.

qRT-PCR detection and quantification. Total RNAs were isolated from cells using TRIzol reagent (Invitrogen Life Technologies, SanDiego, CA, USA). One microgram of RNA was reverse-transcribed to cDNA using the PrimeScript First Strand cDNA synthesis kit (Takara Bio, Inc., Dalian, China) according to the manufacturer's instructions. qRT-PCR was performed on an Applied Biosystems 7500 Real Time PCR system (Applied Biosystems, White Plains, NY, USA). The U6 small RNA and $\beta$ actin mRNA were used as internal controls. All there actions were run in triplicate and the following primers were used: Forward, 5'-AGGAGGTTTTTAATCATCC-3' and reverse, 5'-GTTCCCAGGATGTAAATTA -3' for uc.206; forward, 5'-CGCTTCGGCAGCACATATAC-3' and reverse, 5'-TTCACGAATTTGCGTGTCAT-3' for U6; forward, 5'-CCAGATGAAGCTCCCAGAAT -3' and 5'-TACFTGCAAGTCACAGACTT-3' for P53 mRNA; and forward, 5'-GTCACCAACTGGGACGACAT-3' and reverse,5'-GAGGCGTACAGGGATAGCAC-3' for $\beta$ actin mRNA.

Transfection of cervical cancer cells. Hela and C33A cells were transfected with $10 \mu \mathrm{g}$ relative plasmid or control for
$48 \mathrm{~h}$ before further experiments. The transfected cells were incubated at $37^{\circ} \mathrm{C}$ with $5 \% \mathrm{CO}_{2}$ The uc. 206 and P53 RNA level in the transfected cervical cancer cells were identified by qRT-PCR.

Dual luciferase reporter assay. The full length 3'-UTR of P53 was amplified by PCR from genomic DNA and cloned into the EcoRI and XhaI sites of pGL3-BS vector (Promega, WI, USA). The primers for P53 3'-UTR were as follows: 5'-CATTCTCCACTTCTTGTTCC -3' and 5'-AGATGTGCTTGCAGAATGTA -3'. The mutant construct of P53 3'UTR was generated using a Quick Change mutagenesis kit (Stratagene, Heidelberg, Germany). Co-transfection of reporter vectors and uc.206 mimics or negative control was performed using Lipofectamine 2000 (Invitrogen, San Diego, USA). After $48 \mathrm{~h}$, dual luciferase activity was measured using a dual luciferase reporter assay system according to the manufacturer's protocol (Promega, WI, USA).

CCK8 assay. Cells $\left(5 \times 10^{4} \mathrm{cell} / \mathrm{ml}\right)$ were transfected with uc. 206 or the control vector for $48 \mathrm{~h}$. Then each well was added with $10 \mu \mathrm{l} \mathrm{CCK}-8$ solution, and was incubated for $3 \mathrm{~h}$ at $37^{\circ} \mathrm{C}$ with $5 \% \mathrm{CO}_{2}$. The absorbance was measured at $450 \mathrm{~nm}$ using a microplate reader (Promega, WI, USA).

Cell apoptosis assays. Cells were seeded in 6-well plates and transiently transfected with uc.206 and the control vector. After 48 hours the cells were washed with cold PBS twice and subsequently treated with $5 \mu \mathrm{l}$ of Annexin V and $10 \mu \mathrm{l}$ of propidium iodide (PI) using Alexa Fluor 488 annexin V/Dead Cell Apoptosis Kit (Invitrogen) to determine the cell apoptosis according to the protocol and then analysed by a flow cytometer (FACScalibur, NY, USA).

Western blot analysis. Proteins were extracted by Cell lysis for western kit (Beyotime, Jiangsu, China) according to the instructions. Protein concentration was quantified using Enhanced BCA protein assay kit (Beyotime, Jiangsu, China). For western blot analysis, equal amounts of total protein were boiled, and separated by SDS-PAGE. After electrophoresis, protein was blotted onto a PVDF membrane and blocked for $2 \mathrm{~h}$ at room temperature. Membranes were incubated with anti-P53 antibody (Cell Signaling Technologies, Boston, MA) at 1:1000 dilutions for overnight at $4{ }^{\circ} \mathrm{C}$. P53 protein level was detected by horseradish peroxidase (HRP) conjugated mouse and rabbit secondary antibody (Beyotime, Jiangsu, China) for $2 \mathrm{~h}$ at room temperature. Protein bands were detected on FluorChem FC2 Imaging System (Alpha Innotech, San Leandro, CA).

Statistical analysis. Statistical analyses were performed using SPSS 16.0 software package (SPSS, Chicago, USA). All graphs were created using GraphPad Prism 5 software (GraphPad Software, La Jolla, CA, USA). All data from three independent experiments were expressed as mean \pm SD. Differences were assessed by two-tailed Student's t test. The relationship between P53 and uc.206 expressions was tested with two-tailed Pearson's correlation. $\mathrm{P}<0.05$ was considered statistically significant. 

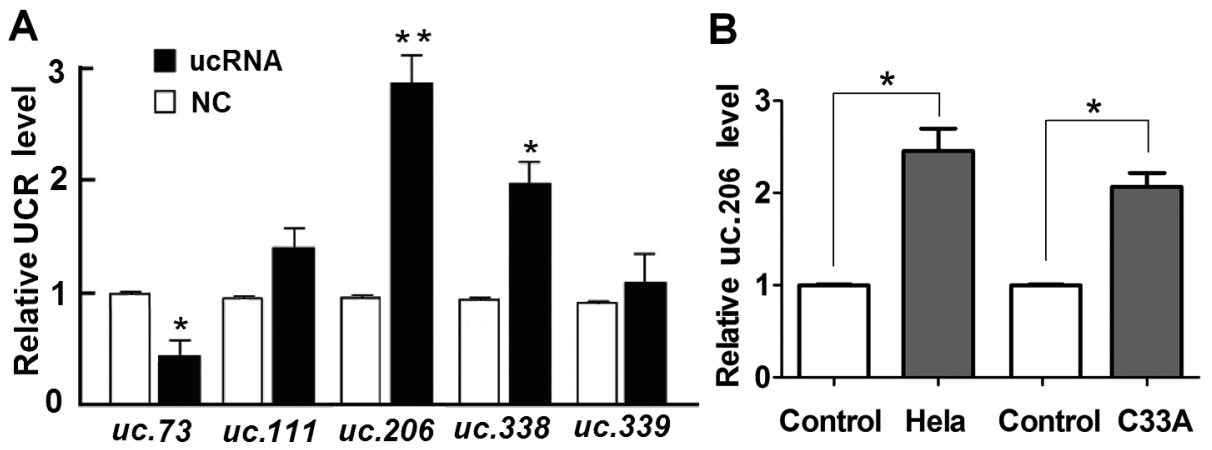

Figure 1. Expression of uc.206 is up-regulated in CC tissues and cell lines. A, expression of uc.206 was up-regulated in $95 \%$ (38 out of 40 ) of CC tissues compared with the adjacent normal cervical tissues $\left({ }^{* *} \mathrm{p}<0.01, \mathrm{n}=40\right)$. B, expression of uc.206 was much more in Hela and C33A cell lines than that of human cervical tissue cell $\left({ }^{*} \mathrm{p}<0.05, \mathrm{n}=3\right)$.

Results

Expression of uc.206 is up-regulated in CC tissues and cell lines. Expression profiles of five UCRs (uc.73, uc.111, uc.206, uc.338, and uc.339) previously shown to involved in tumorigenesis $(6,8)$ were detected by qRT-PCR in 40 pairs of CC tissues and matched nomal cervical tissues. Among the five, uc.206 showed significant up-regulation in group of CC tissues versus matched group of nomal tissues $\left({ }^{*} \mathrm{p}<\right.$ 0.01, Figure 1A). We also found that expression of uc.206 was much more in two cervical cancer cell lines than that of human cervical tissue cell $\left({ }^{\star} \mathrm{p}<0.05\right.$, Figure $\left.1 \mathrm{~B}\right)$. Collectively, the above findings suggest that excess of uc.206 expression may play an important role in tumorigenesis of cervical carcinoma.
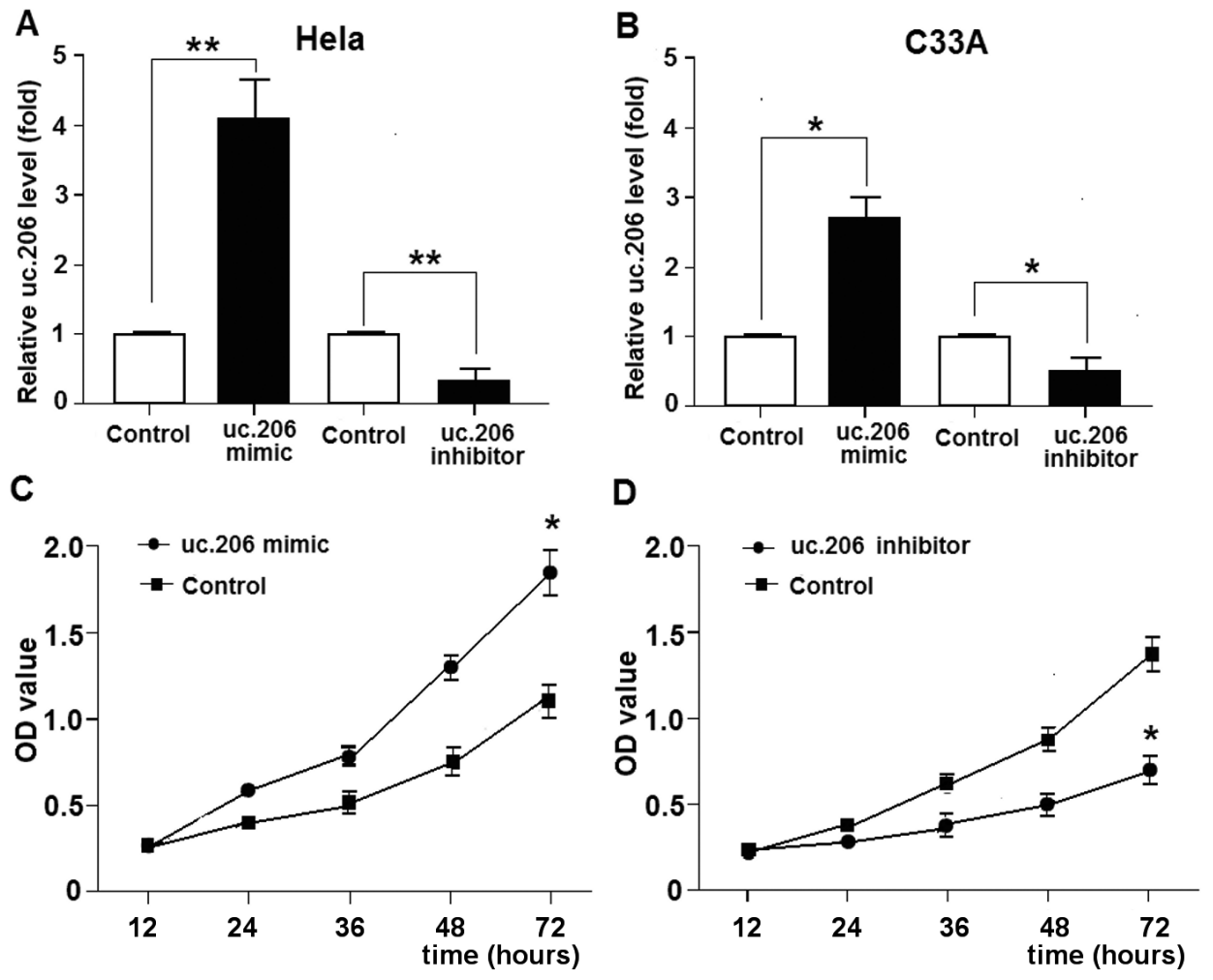

Figure 2. Upregulation of uc.206 promotes cell proliferation in CC cells. A, B, Uc.206 was significantly upregulated in the cells expressing the mimic and significantly downregulated in the cells transfected with the inhibitor $\left({ }^{*} \mathbf{p}<0.05, \mathrm{n}=3\right)$. The Hela cells exhibited higher transfection efficiency than the C33A cells. C, Cell proliferation of Hela transfected with the uc.206 mimic was measured by CCK8 analysis $\left({ }^{\star} p<0.05, n=3\right)$. D, Hela cells transfected with the uc.206 inhibitor were analysed for cell proliferation via CCK8 analysis $\left({ }^{*} p<0.05, n=3\right)$. 


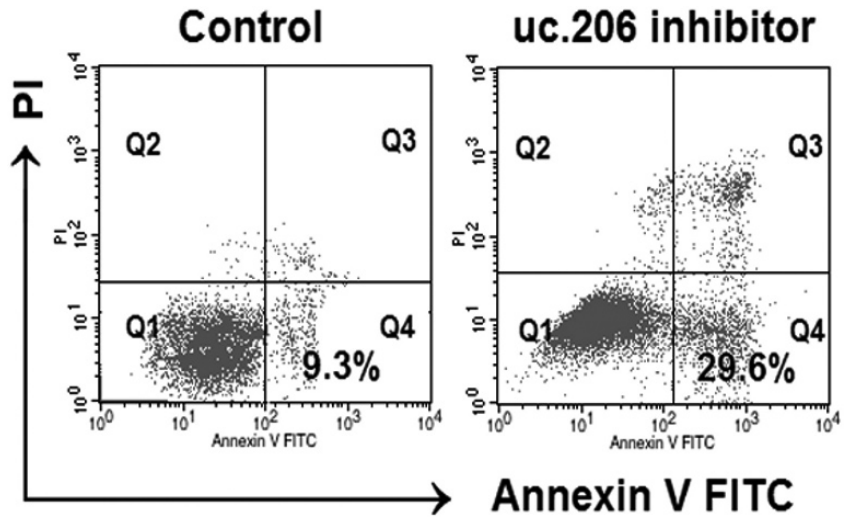

Figure 3. Upregulation of uc.206 inhibits cell apoptosis in CC cells. After transfection with the uc.206 inhibitor, Hela cell apoptosis was measured by flow cytometry. Knockdown of uc.206 demonstrated a significantly increase $(29.6 \%)$ in apoptosis percent when compared to controls $(9.3 \%)$ $(\mathrm{p}<0.05, \mathrm{n}=3)$.

Upregulation of uc.206 promotes cell proliferation and inhibits cell apoptosis in CC cells. To explore the biological function of uc.206 in CC tissue, the chemically synthesized uc.206 mimic and inhibitor were transfected into the Hela and C33A CC cell lines to up-regulate and down-regulate uc.206 expression, respectively. Transfection efficiency was evaluated via qRT-PCR and is shown in Figure 2A and 2B. The Hela cell line exhibited higher transfection efficiency than C33A cells; therefore, all of our experiments in this study were conducted in the Hela cell line. To detect the effect of uc.206 on proliferation of Hela cells, we performed CCK8 assays on the transfected Hela cells. Our data showed that over-expression of uc.206 led to a statistically significant increase in Hela cell proliferation compared to the negative control cells $(\mathrm{p}<0.05$, Figure 2C). Conversely, the proliferation rate of the Hela cells treated with the uc.206 inhibitor was decreased when compared to control cells $(\mathrm{p}<0.05$, Figure 2D).

Next, we analyzed apoptosis in the transfected Hela cells via flow cytometry. As shown in Figure 3, Hela cells transfected with the uc.206 inhibitor demonstrated a significantly increase in apoptosis percent when compared to controls $(\mathrm{p}<0.05)$. Thus, uc.206 expression may be upregulated in Hela cells and CC tissues because expression of this UCR promotes proliferation and inhibits apoptosis in Hela cells.

P53 is a direct target of uc.206. According to bioinformatic prediction by http://blast.ncbi.nlm.nih.gov/ and http://www. noncode.org/NONCODERv3, we tried identifying potential targets for uc.206 and found the gene P53 as the putative target gene for uc.206. To verify whether uc.206 directly targeted P53, Duel luciferase reporter assays were conducted. As shown in Figure 4, co-transfection of 293T cells with P53-3'UTR/ pGL3-BS and uc.206 caused significant decrease in the luciferase activity compared with the negative control $(\mathrm{p}<0.05)$. This repressive effect was disappeared by point mutations in the binding sites of the P53-3'UTR. This result indicated that uc. 206 exerts inhibitory effects on P53 expression via interaction with the 3'UTR of P53.

Uc.206 negatively regulates expression of P53. To further confirm that P53 is a target gene for uc.206, RT-PCR and Western blot analysis were used to detect the expression of P53 by uc.206 mimic or inhibitor in Hela cells. Our data showed that up-regulated expression of P53 via uc.206 inhibitor (Figure $5 \mathrm{~A}$ ) and down-regulated expression of P53 via uc.206 mimic (Figure 5B) compared with control. Taken together, our results
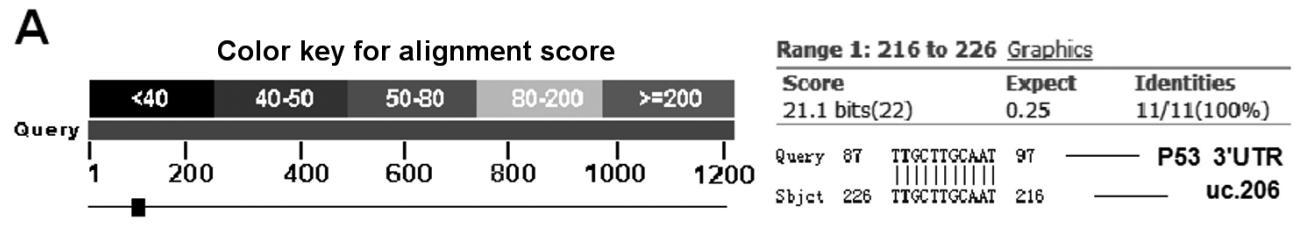

B

Reporter Vector
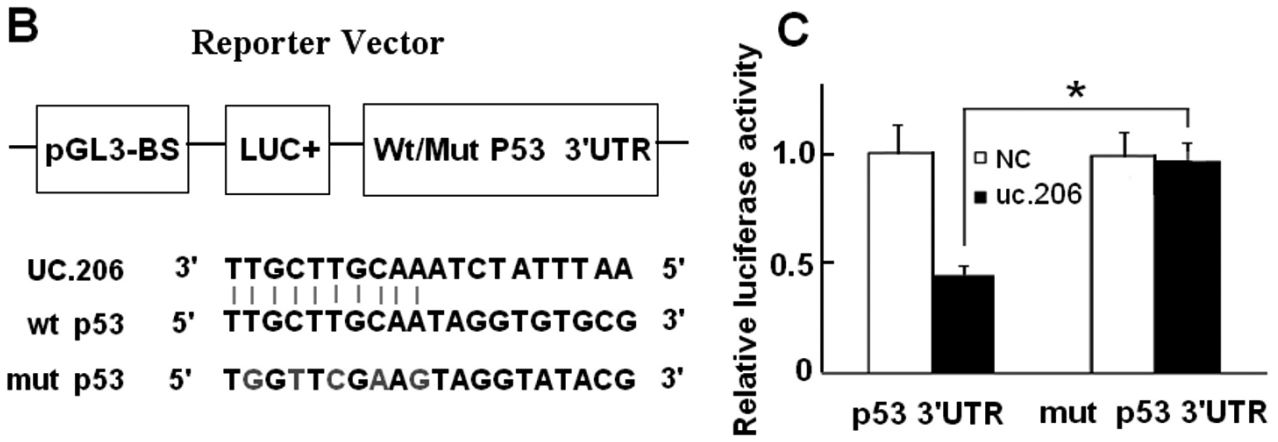

Figure 4. P53 is a direct target of uc.206. A, P53 was the target gene of uc.206 by blast.ncbi.nlm.nih.gov prediction. B, Diagram of the luciferase reporter plasmids with the wild-type or mutant P53 3'UTR. C, The relative luciferase activity in Hela cells was determined after the plasmid with wild-type or mutant P53 3'UTR was co-transfected with P53. Data are presented as mean \pm SD. Two-tailed Student's t test was used to analyze the significant differences, ${ }^{*} \mathrm{p}<0.05, \mathrm{n}=3$. 
A

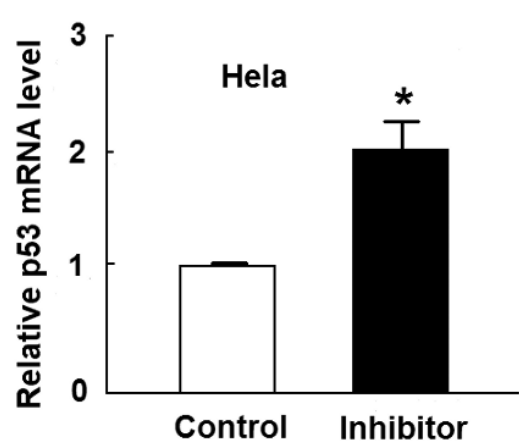

P53

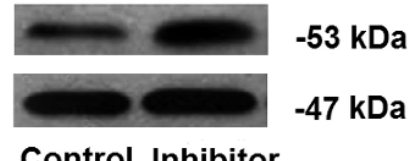

B

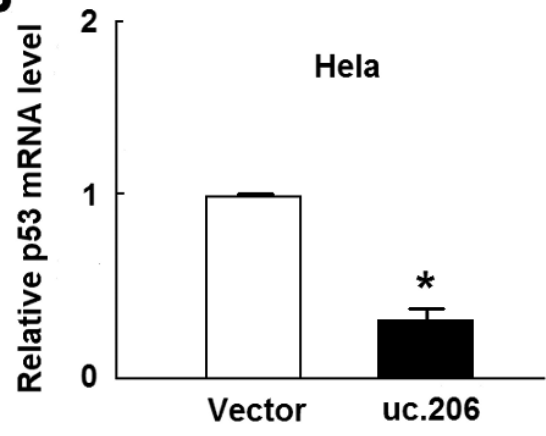

P53

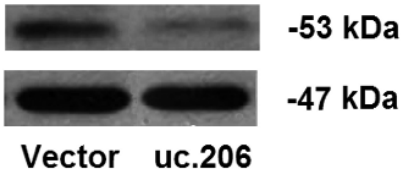

Figure 5. Uc.206 negatively regulates expression of P53. mRNA and protein levels of P53 were measured by qRT-PCR and Western blotting. Hela cells were transfected with the uc.206 inhibitor or mimic for $48 \mathrm{~h}$. A, Expression of P53 was significantly upregulated at the mRNA and protein level after uc.206 inhibitor compared with control $\left({ }^{*} \mathrm{p}<0.05, \mathrm{n}=3\right)$. B, Expression of P53 was notablely downregulated at the mRNA and protein level after uc.206 mimic compared with empty vector $\left({ }^{*} \mathrm{p}<0.05, \mathrm{n}=3\right)$.

suggest that uc.206 negatively regulates P53 gene expression at posttranscriptional level, and P53 is a potential target gene of uc.206.

Uc. 206 was negatively correlated with P53 in the CC tissue. To assess the correlation of the uc. 206 and P53 in cervical carcinoma, we detected the RNA levels of uc.206 and P53 in cervical carcinoma and adjacent normal cervical tissues by using qRT-PCR. As shown in Figure 6, when the P53 mRNA levels were plotted against uc.206 expression, a significant inverse correlation was obtained by two-tailed Pearson's correlation analysis $(\mathrm{r}=-0.625 ; \mathrm{p}<0.05)$.

Knock down P53 in Hela cells promotes cell proliferation and inhibits cell apoptosis. To confirm the effects of P53 on the proliferation and apoptosis of Hela cell, P53 expression was knocked down by a P53 shRNA (sh-P53). The mRNA and protein expression of P53 was significantly down-regulated in Hela cell after P53 shRNA treatment (Figure 7A). Consistently, the knockdown of P53 strongly promotes cell proliferation and inhibits cell apoptosis in Hela cells, which resembled the over-expression effects of uc.206 (Figure 7B, 7C). These data suggest that knockdown of P53 promotes cell proliferation and inhibits cell apoptosis in CC cell and P53 is an effective target gene of uc. 206 .

Overexpression of P53 rescues the effect of uc. 206 overexpression in Hela cells. To verify the effects of P53 overexpress in uc. 206 overexpression of Hela cells, P53 gene was overexpressed via migRI-P53-GFP plasmid. The mRNA and protein expression of P53 was dramatically upregulated in Hela cells after P53 treatment (Figure 8A), compared with empty plasmid control. Invariably, the overexpression of P53 remarkably inhibited cell proliferation and promoted cell apoptosis

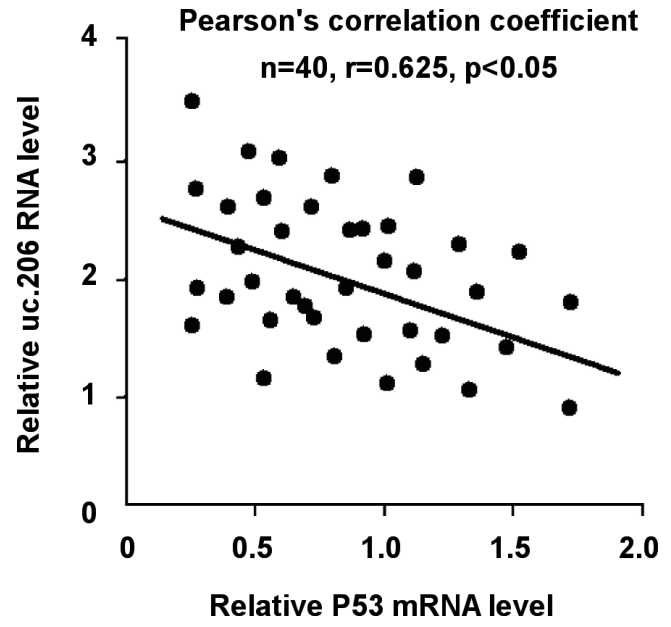

Figure 6. Uc.206 was negatively correlated with P53 in the CC tissue. Uc.206 was negatively correlated with P53 at RNA levels in the CC tissue compared with control by two-tailed Pearson's correlation analysis $(\mathrm{r}=-0.625 ; \mathrm{p}<0.05, \mathrm{n}=40)$.

(Figure 8B, 8C). These data suggest that upregulation of P53 rescues the effect of uc. 206 overexpression in Hela cells.

\section{Discussion}

A non-coding RNA (ncRNA) is a functional RNA molecule that is not translated into a protein. NcRNA include microRNA, long non-coding RNA (lnc-RNA), ultraconserved regions(UCRs) and et al. UCRs are non-coding gene 

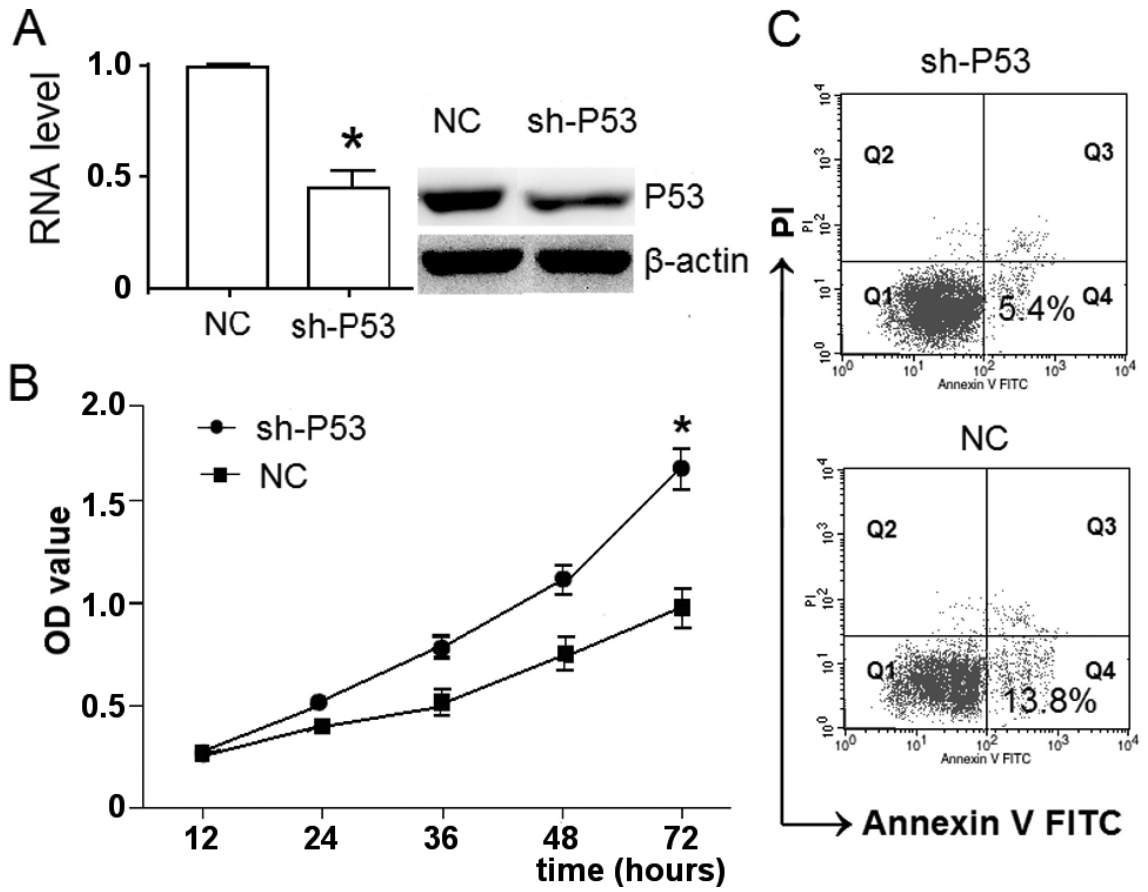

Figure 7. Knock down P53 in Hela cells regulates proliferation and apoptosis. A, Transfection of P53 shRNA inhibited the mRNA and protein expression of P53 in Hela cells Knockdown of Rac1 by shRNA promotes cell proliferation (B) and inhibits cell apoptosis (C) in Hela cells, which resembled the over-expression effects of uc.206. Data are presented as the mean \pm standard deviation. Two-tailed Student's t-test was used to analyze the significant differences. ${ }^{\star} \mathbf{P}<0.05, \mathrm{n}=3$, vs. the corresponding control.

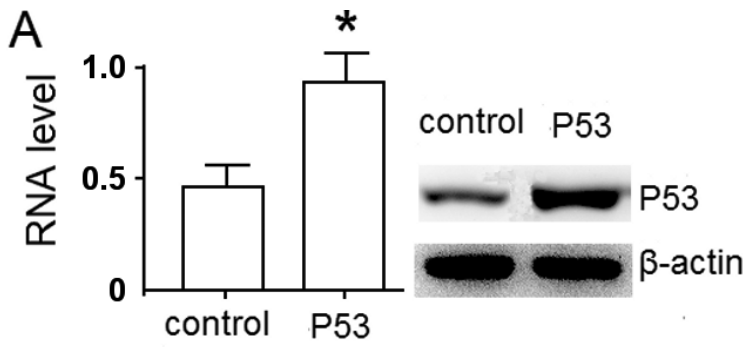

C
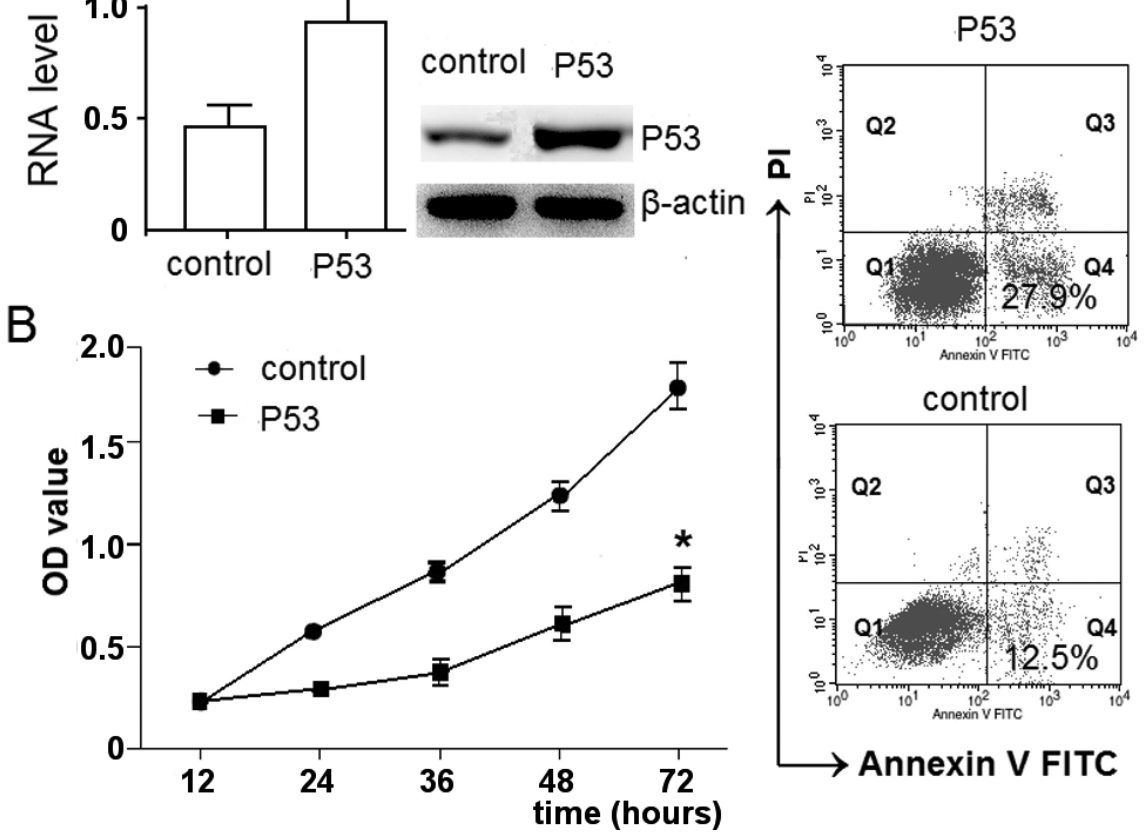

Figure 8. Overexpression of P53 rescues the effect of uc. 206 overexpression in Hela cells. A, Transfection of migRI-P53-GFP plasmid upregulated the mRNA and protein expression of P53 in Hela cells. Overexpression of P53 inhibits cell proliferation (B) and promotes cell apoptosis (C) in uc.206 overexpression of Hela cells. Data are presented as the mean \pm standard deviation. Two-tailed Student's t-test was used to analyze the significant differences. ${ }^{*} \mathrm{P}<0.05, \mathrm{n}=3$, vs. the corresponding control. 
sequences with strict conserved across among mice, rats, and humans. It belongs to part of non-protein-coding DNA and regulates expression and translation of mRNAs. By regulating protein production post-transcriptionally, many UCRs act as oncogenes or tumor suppressor genes $(6,9-12)$. However, the regulation of uc.206 and its precise mechanisms of action in cervical cancers are not known.

P53, a tumor suppressor, inhibited cell proliferation by arresting cell cycle and inducing apoptosis in response to cellular stress $[13,14]$. Disruption of P53 levels could promote tumor progression and inhibit apoptosis, but elevate the DNA damage $[13,15]$. Previous report suggested that ncRNA could regulate P53 protein production post-transcriptionally. For example, anta-miR-150 treated tumor tissue, the expression of P53 is increased, and indicated P53 is the potential downstream target of miR-150 but the direct evidences were not provided [16]. By using luciferase reporter system, we verified miR-150 directly target P53 transcript at its 3'UTR and inhibit its translation [17]. overexpression of MEG3(a non-coding RNA) significantly stabilized p53 expression which leads to an increased level of P53 protein. Therefore, it is likely that MEG3 activates P53 through inhibition of P53 ubiquitination and blockage of P53 degradation [18]. Y zhou et al. have found that MDM2 expression was repressed by MEG3, suggesting that down-regulation in MDM2 protein levels contributes to P53 activation by MEG3 [19].

In this study, we screened for 5 UCRs in 40 pair of CC patients and found that the RNA levels of uc.206 were significantly upregulated in CC tissues. We also found that the upregulation of uc. 206 had negative correlation with mRNA levels of P53. By using dual luciferase reporter system, we found that P53 is directly targeted by uc.206. Over-expression of uc. 206 promoted cell proliferation and inhibition of uc.206 suppressed cell proliferation and promoted apoptosis in Hela and C33A cells. Knockdown of P53 promotes cell proliferation and inhibits cell apoptosis in CC cell and overexpression of P53 in uc. 206 overexpression of Hela cells remarkably inhibited cell proliferation and promoted cell apoptosis.

In summary, these data illustrate the possible role of uc.206 and P53 expression in the pathogenesis of CC, uc.206 may be an important oncogene in cervical carcinoma, and uc.206 might be helpful in clinical diagnosis and therapy for preventing cervical cancer.

Acknowledgements: This work was supported by Health department of Jiangsu province Science Foundation (Grant No. JZ201310) and 2012-2015 "Cyanine Project" Foundation in Jiangsu province colleges and universities.

\section{References}

[1] JEMAL A, BRAY F, CENTER MM, FERLAY J, WARD E et al. Global cancer statistics. CA Cancer J Clin 2011; 61: 69-90. http://dx.doi.org/10.3322/caac.20107

[2] KENT A. „HPV Vaccination and Testing.“. Reviews in obstetrics and gynecology. 2010; $3: 33-34$.
[3] KANAYAMA T, MABUCHI S, SHIMURA K, HISAMATSU T, ISOHASHI $\mathrm{F}$ et al. Prognostic factors for survival in cervical cancer patients with bone metastasis.Eur J Gynaecol Oncol. 2015; 36: 290-293.

[4] BEJERANO G, LOWE CB, AHITUV N, KING B, SIEPEL A et al. A distal enhancer and an ultraconserved exon are derived from a novel retroposon. Nature. 2006; 441: 87-90. http:// dx.doi.org/10.1038/nature04696

[5] KATZMAN S, KERN AD, BEJERANO G, FEWELL G, FULTON L et al. Human genome ultraconserved elements are ultraselected.Science. 2007; 317: 915. http://dx.doi. org/10.1126/science. 1142430

[6] CALIN GA, LIU CG, FERRACIN M, HYSLOP T, SPIZZO R et al. Ultraconserved regions encoding ncRNAs are altered in human leukemias and carcinomas. Cancer Cell. 2007; 12: 215-229. http://dx.doi.org/10.1016/j.ccr.2007.07.027

[7] LUJAMBIO A, PORTELA A, LIZ J, MELO SA, ROSSI S et al. CpG island hypermethylation-associated silencing of noncoding RNAs transcribed from ultraconserved regions in human cancer. Oncogene. 2010; 29: 6390-6401. http://dx.doi. org/10.1038/onc.2010.361

[8] SANA J, HANKEOVA S, SVOBODA M, KISS I, VYZULA R et al. Expression levels of transcribed ultraconserved regions uc. 73 and uc. 388 are altered in colorectal cancer. Oncology. 2012; 82: 114-118. http://dx.doi.org/10.1159/000336479

[9] BRACONI C, VALERI N, KOGURE T,GASPARINI P, HUANG $\mathrm{N}$ et al. Expression and functional role of a transcribed noncoding RNA with an ultraconservedelement in hepatocellular carcinoma.Proc Natl Acad Sci U S A. 2011; 108: 786-791. http://dx.doi.org/10.1073/pnas.1011098108

[10] WANG C, YAN G, ZHANG Y, JIA X, BU P. Long non-coding RNA MEG3 suppresses migration and invasion of thyroid carcinoma by targeting of Rac1. Neoplasma. 2015; 62: 541-549. http://dx.doi.org/10.4149/neo 2015065

[11] MESTDAGH P, FREDLUND E, PATTYN F, RIHANI A,VAN MAERKEN T et al. An integrative genomics screen uncovers ncRNA T-UCR functions in neuroblastoma tumours. Oncogene. 2010; 29: 3583-3592. http://dx.doi.org/10.1038/ onc.2010.106

[12] CATUCCI I, VERDERIO P, PIZZAMIGLIO S, MANOUKIAN $\mathrm{S}$, PEISSEL B et al. SNPs in ultraconserved elements and familial breast cancer risk. Carcinogenesis. 2009; 30: 544-545. http://dx.doi.org/10.1093/carcin/bgn289

[13] BENCHIMOL S. P53-dependent pathways of apoptosis. Cell Death Differ. 2001; 8: 1049-1051. http://dx.doi.org/10.1038/ sj.cdd. 4400918

[14] SHEN Y, WHITE E. P53-dependent apoptosis pathways. Adv. Cancer Res. 2001; 82: 55-84. http://dx.doi.org/10.1016/ $\underline{\text { S0065-230X(01)82002-9 }}$

[15] LI Y, ZHAO Y, JIANG R, XU Y, LING M et al. DNAPKcs-mediated stabilization of p53 by JNK2 is involved in arsenite-induced DNA damage and apoptosis in human embryo lung fibroblast cells. Toxicol Lett. 2012; 210: 302-310. http://dx.doi.org/10.1016/j.toxlet.2012.02.006

[16] LI Y.J., ZhANG Y.X., WANG P.Y., ChI Y.L., ZhANG, C et al.Regression of A549 lung cancer tumors by anti-miR-150 vector. Oncol. Rep. 2012; 27: 129-134. 
[17] NI ZhANG, XIANG WEI, LIJUN XU. miR-150 promotes the proliferation of lung cancer cells by targeting P53. FEBS Letters. 2013; 587: 2346-2351. http://dx.doi.org/10.1016/j. febslet.2013.05.059

[18] ZHU J, LIU S, YE F, SHEN Y, TIE Y et al. Long Noncoding RNA MEG3 Interacts with p53 Protein and Regulates Partial p53 Target Genes in Hepatoma Cells. PLoS One. 2015; 10: e0139790. http://dx.doi.org/10.1371/journal.pone.0139790

[19] ZHOU Y, ZHONG Y, WANG Y, ZHANG X, BATISTA DL et al. Activation of p53 by MEG3 non-coding RNA. J Biol Chem. 2007; 282: 24731-42. http://dx.doi.org/10.1074/jbc. M702029200 